\section{Case Reports in Oncology}

\title{
Transurethral Resection for the Treatment of an Inflammatory Myofibroblastic Tumor of the Urinary Bladder: A Case Report
}

\author{
Kamel Fadaak Aali Al-Otaibi Abdullah Al-Zahrani Abdullah Alhaam \\ Omran Al-Dandan Haitham Kussaibi Osama Al-Sowayan \\ Reem Aldamanhori
}

Imam Abdulrahman Bin Faisal University, Dammam, Saudi Arabia

\section{Keywords}

Bladder tumor - Inflammatory myofibroblastic tumor - Transurethral resection of bladder tumor

\begin{abstract}
Inflammatory myofibroblastic tumors (IMT) of the urinary bladder is a remarkably rare bladder tumor. To this day, no standardized treatment protocol has been recognized. Here we report a case of bladder IMT in a 14-year-old girl presenting with urgency, frequency, and gross painless hematuria for a week. Complete excision of the bladder IMT was amenable with transurethral resection of bladder tumor (TURBT). Follow-up cystoscopy did not detect any recurrence. Minimally invasive bladder-sparing treatments are a valid option for treating IMT of the bladder.

\section{Introduction}

Inflammatory myofibroblastic tumor (IMT) of the bladder is a rare disease with no recognized recommendation in treatment. IMT has been termed as plasma cell granuloma,

\begin{tabular}{ll}
\hline & Reem Aldamanhori \\
Urology, Imam Abdulrahman Bin Faisal University \\
Imam Abdulrahman Bin Faisal Campus \\
Dammam 34211 (Saudi Arabia) \\
E-Mail rdamanhori@iau.edu.sa
\end{tabular}




\section{Case Reports in Oncology}

Case Rep Oncol 2019;12:344-353

DOI: $10.1159 / 000500503$

(c) 2019 The Author(s). Published by S. Karger AG, Basel www.karger.com/cro

Fadaak et al.: Transurethral Resection for the Treatment of an Inflammatory Myofibroblastic Tumor of the Urinary Bladder: A Case Report

inflammatory pseudotumor, pseudomalignant spindle cell proliferation, or pseudosarcomatous myofibroblastic tumor [1]. The diversity in terminology created misperceptions and struggles in generating a unified standardized treatment for this entity. Owing to its rarity, a lack in single center experience has developed.

Inflammatory myofibroblastic tumor (IMT) was first described by Roth [2] in 1980. It is defined as a proliferative lesion arising from submucosal stroma, of low or indeterminate malignant potential [3]. Although rare, comprising less than $1 \%$ of all bladder tumors, the bladder is the most common site involved in the genitourinary tract.

Systemic symptoms such as fever and weight loss are typically absent for tumors in the genitourinary tract. Although presentation may include dysuria and pelvic pain, the most common presenting symptom for IMT in the bladder is painless hematuria [4]. The treatment decision is highly individualized, options include transurethral resection of bladder tumor (TURBT), partial cystectomy, and/or radical cystectomy.

\section{Case Presentation}

A 14-year-old girl presented to the Urology clinic with storage symptoms mainly urgency and frequency. She also had recurrent attacks of gross painless hematuria for about 1-week duration. She had negative history of urinary tract infections (UTIs), surgical procedures, urethral instrumentation. There was no positive family history of malignancy, neither the patient or any member of the family smoked. Systemic review and physical examination were unremarkable. Initial investigations included urine microscopy, which demonstrated red blood cells (RBCs) of more than 100 per high power field. Other parameters of the urine microscopy were within normal ranges. Urine culture showed no growth, and urine cytology was negative for malignant cells.

Imaging was warranted, and a urinary tract ultrasound (US) showed an immobile lesion, seen at the right lateral wall of the bladder measuring $(2 \times 1.5 \mathrm{~cm})$ with evidence of internal vascularity on Doppler ultrasound (Fig. 1). She was sent for Computed Tomography Urography (CTU), which revealed an asymmetrical wall thickening notable at the right posterolateral aspect of the bladder. Within this, a well-defined intramural focal hyper-enhancing lesion is seen with central area of hypoattenuation (ring enhancement). The overlying urothelium was intact. There was no extramural extension of disease, no peri vesical fat stranding or free fluid, and no regional lymph nodes (Fig. 2).

She underwent cystoscopy and TURBT (Fig. 3) and the pathology revealed Inflammatory myofibroblastic tumor of the urinary bladder. Microscopic examination of the lesion showed submucosal spindle cells proliferation arranged in a fascicular and storiform pattern (Fig. 4) on a myxoid background with mixed inflammatory cells infiltrate (Fig. 5) composed mainly of lymphocytes, plasma cells along with scattered eosinophils. The lesion abuts on adjacent muscularis propria. Tumor cells frequently have ovoid nuclei with prominent nucleoli (Fig. 6). Mitoses are rare. The Immunohistochemical study (Fig. 7) revealed tumor cells that are diffusely positive for SMA (smooth muscle actin), ALK1 and P-catenin (cytoplasmic), Ki67 5\%. Tumor cells are negative for desmin, CD34 and c-kit.

The patient is a minor. Written informed consent was taken from the patient and her guardian (father) to publish the case. Consent also included approval of publication of the images, and pathology slides without mentioning the patients name or medical number or any other identifier to the identity of this patient.

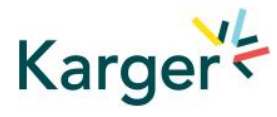




\section{Case Reports in Oncology}

\section{Discussion}

Inflammatory myofibroblastic tumor (IMT) rarely involves the urinary bladder. A systematic review by Teoh et al. [5] in 2014 evaluated 182 cases of IMT of the urinary bladder in literature. Another literature review by Song et al. [1] in 2019 revealed 16 more patients, for a total of 198 cases of IMT of the urinary bladder to date. Frequency and urgency (irritative symptoms) along with hematuria are the most common clinical manifestations [4]. The hematuria can be so severe that some patients have profound anemia on presentation [5].

Liang et al. [6] described a certain appearance of IMT of the urinary bladder on CT scans. He describes it as lesions that appeared mainly on the anterior wall of the bladder, with ring enhancement on the contrast-enhanced CT. Although this was evident in our case, being nonspecific and inconclusive, radiological examinations including CT with contrast is insufficient to establish a diagnosis. The main diagnostic tool is tissue biopsy via TURBT and histopathological examination.

IMT of the bladder are considered benign, with local tumor recurrence rate as low as $4 \%$ [1]. Transurethral resection of bladder tumor (TURBT) is often performed, a partial cystectomy option is chosen according to the histopathological result. Although IMT of the bladder are considered a benign entity, with local extension and recurrence being an infrequent outcome, controversy arises regarding their metastatic potential. Only two cases in the literature have reported metastasis of bladder IMT [3, 7]. In one case it occurred post local radiation. And in the other case [7] infiltration of the IMT tumor cells through the muscularis propria of the bladder and into the peri vesical fat was seen on the cystectomy sample, the patient had local extension to the adjacent bowel rather than distant metastasis.

\section{Conclusion}

IMT of the bladder is rare with multiple differential diagnoses. Thus, histopathological data is anticipated to distinguish IMT from malignant diseases. Considering its benign nature and low risk of local recurrence, delay in histopathologic diagnosis may result in excessive treatment for IMT. Treatment is individualized yet a bladder-preserving minimally-invasive approach such as TURBT is preferable. procedures such as partial cystectomy may be required depending on the variation in histology. Upcoming studies should concentrate on developing urine markers or other diagnostic methods to speed up diagnosis and treatment.

\section{Statement of Ethics}

The authors have no ethical conflicts to disclose.

\section{Disclosure Statement}

The authors have no conflicts of interest to declare.

\section{Karger'}




\section{Case Reports in Oncology}

\begin{tabular}{l|l}
\hline Case Rep Oncol 2019;12:344-353 \\
\hline DOI: 10.1159/000500503 & $\begin{array}{l}\text { @ 2019 The Author(s). Published by S. Karger AG, Basel } \\
\text { www.karger.com/cro }\end{array}$ \\
\hline
\end{tabular}

Fadaak et al.: Transurethral Resection for the Treatment of an Inflammatory Myofibroblastic Tumor of the Urinary Bladder: A Case Report

\section{References}

1 Song D, Jiao W, Gao Z, Liu N, Zhang S, Zong Y, et al. Inflammatory myofibroblastic tumor of urinary bladder with severe hematuria: A Case report and literature review. Medicine (Baltimore). 2019 Jan;98(1):e13987.

2 Roth JA. Reactive pseudosarcomatous response in urinary bladder. Urology. 1980 Dec;16(6):635-7.

3 Montgomery EA, Shuster DD, Burkart AL, Esteban JM, Sgrignoli A, Elwood L, et al. Inflammatory myofibroblastic tumors of the urinary tract: a clinicopathologic study of 46 cases, including a malignant example inflammatory fibrosarcoma and a subset associated with high-grade urothelial carcinoma. Am J Surg Pathol. 2006 Dec;30(12):1502-12.

4 Patnana M, Sevrukov AB, Elsayes KM, Viswanathan C, Lubner M, Menias CO. Inflammatory pseudotumor: the great mimicker. AJR Am J Roentgenol. 2012 Mar;198(3):W217-27.

5 Teoh JY, Chan NH, Cheung HY, Hou SS, Ng CF. Inflammatory myofibroblastic tumors of the urinary bladder: a systematic review. Urology. 2014 Sep;84(3):503-8.

6 Liang W, Zhou X, Xu S, Lin S. CT Manifestations of Inflammatory Myofibroblastic Tumors (Inflammatory Pseudotumors) of the Urinary System. AJR Am J Roentgenol. 2016 Jun;206(6):1149-55.

7 Libby EK, Ellis LT, Weinstein S, Hammer RD, Murray KS. Metastatic inflammatory myofibroblastic tumor of the bladder. Urol Case Rep. 2018 Nov;23:10-2.
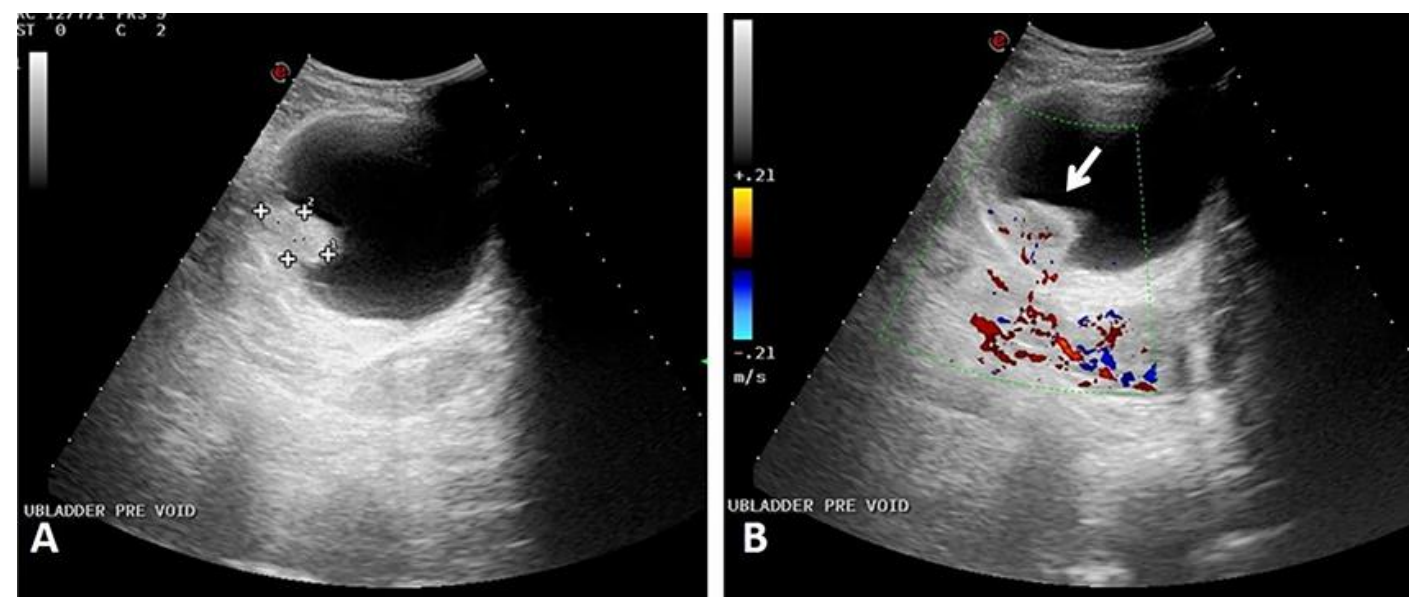

Fig. 1. Ultrasound of urinary bladder, grey scale (A), and color doppler (B) images. Note the echogenic lesion within the wall (measurement cursor, in A), protruding into the lumen. It demonstrates internal vascularity seen as intralesional color (arrow in B). 


\section{Case Reports in Oncology}

\begin{tabular}{l|l}
\hline Case Rep Oncol 2019;12:344-353 \\
\hline DOI: 10.1159/000500503 & $\begin{array}{l}\text { @ 2019 The Author(s). Published by S. Karger AG, Basel } \\
\text { www.karger.com/cro }\end{array}$ \\
\hline
\end{tabular}

Fadaak et al.: Transurethral Resection for the Treatment of an Inflammatory Myofibroblastic Tumor of the Urinary Bladder: A Case Report
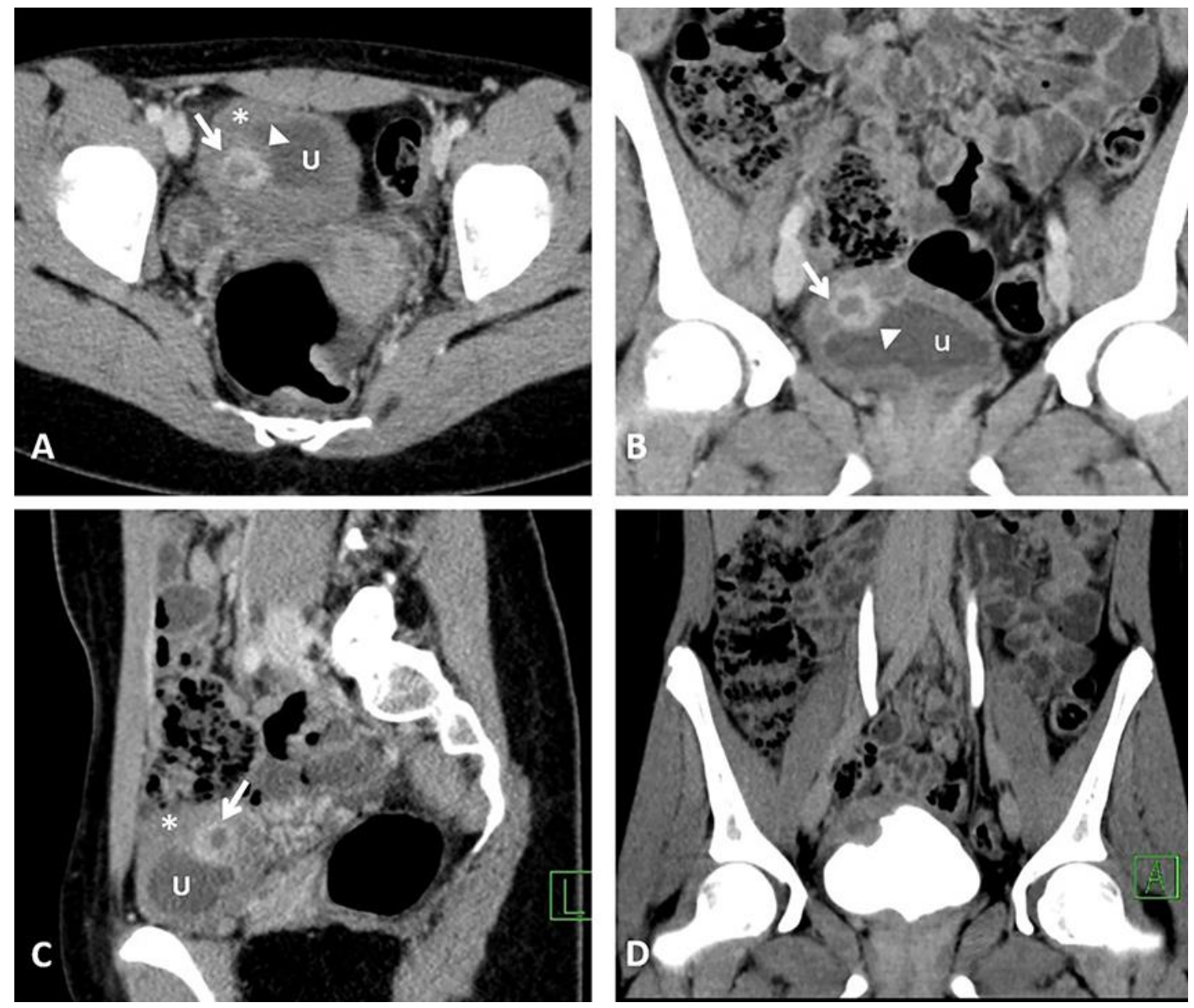

Fig. 2. CT urogram axial (A), coronal (B), and sagittal (C) in nephrographic phase, and coronal (D) in execratory phase. Wall thickening $\left({ }^{*}\right)$ in $\mathbf{A}-\mathbf{C}$, at the right posterolateral aspect of bladder dome, within which a hyper-enhancing focal lesion (white arrow) is seen. The lesion bulges into the bladder lumen (U) but intact overlying mucosa (arrowhead). 
Case Reports in Oncology
Case Rep Oncol 2019;12:344-353 DOI: $10.1159 / 000500503$ (c) www.karger.com/cro

Fadaak et al.: Transurethral Resection for the Treatment of an Inflammatory Myofibroblastic Tumor of the Urinary Bladder: A Case Report
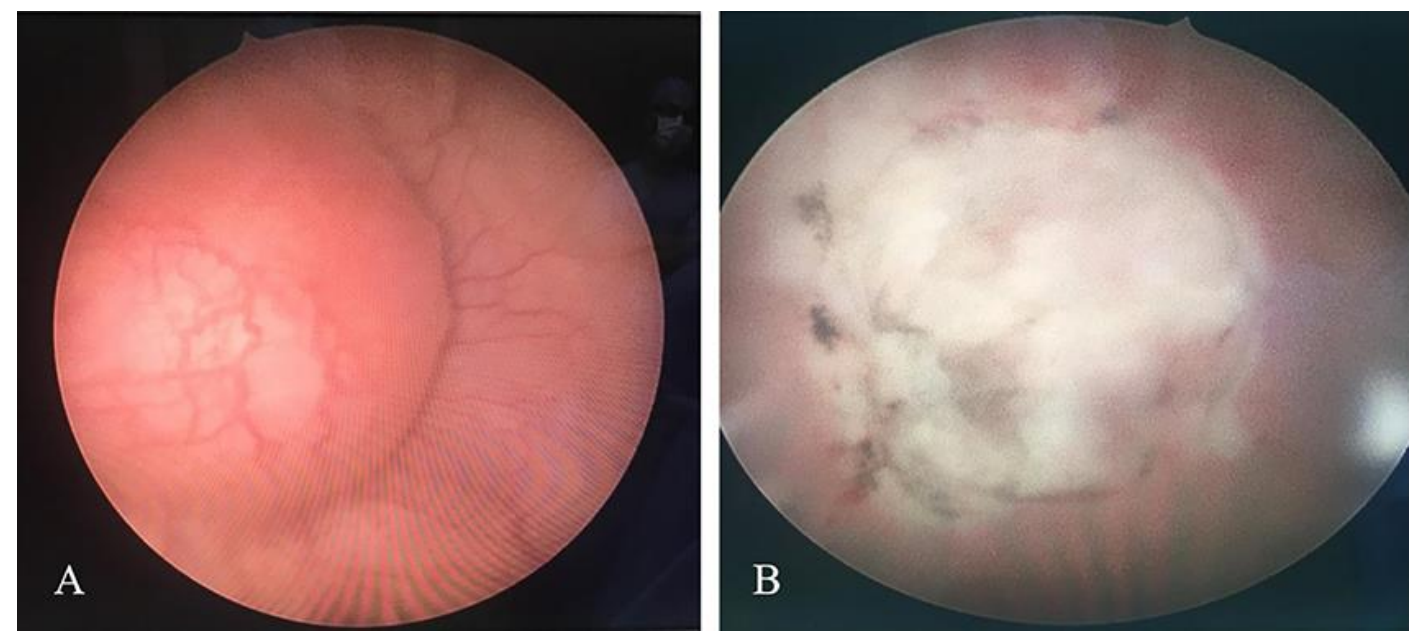

Fig. 3. A Cystoscopic appearance of the bladder mass which obviously has an intact mucosa gives a hint of submucosal origin. B Cystoscopic appearance post resection. 


\section{Case Reports in Oncology}

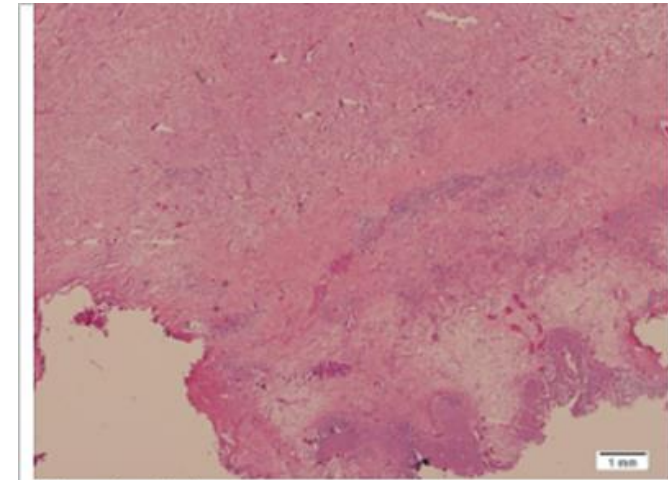

Fig. 4a (5x)

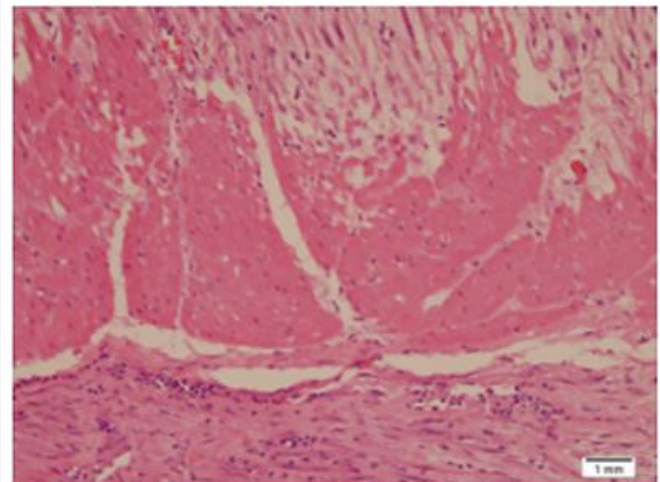

Fig. 4c (10x)

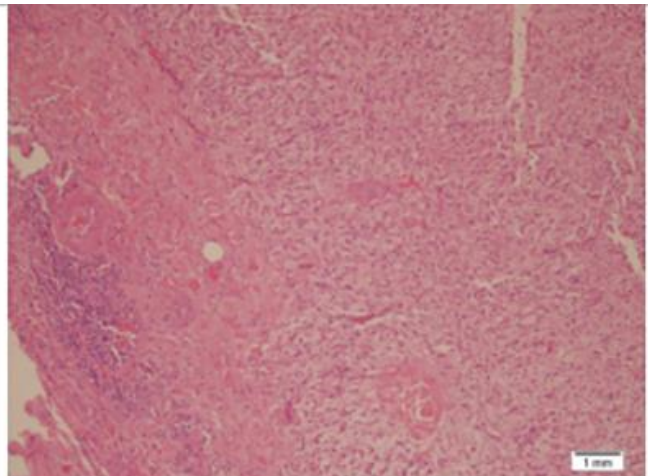

Fig. 4b (10x)

Fig. 4. Microscopic examination of the lesion showed submucosal spindle cells proliferation arranged in a fascicular and storiform pattern (Fig. 4) on a myxoid background with mixed inflammatory cells infiltrate (Fig. 5) composed mainly of lymphocytes, plasma cells along with scattered eosinophils. The lesion abuts on adjacent muscularis propria. Tumor cells frequently have ovoid nuclei with prominent nucleoli (Fig. 6). Mitoses are rare. The Immunohistochemical study (Fig. 7) revealed tumor cells that are diffusely positive for SMA (smooth muscle actin), ALK1 and P-catenin (cytoplasmic), Ki67 5\%. Tumor cells are negative for desmin, CD34 and c-kit. 


\section{Case Reports in Oncology}

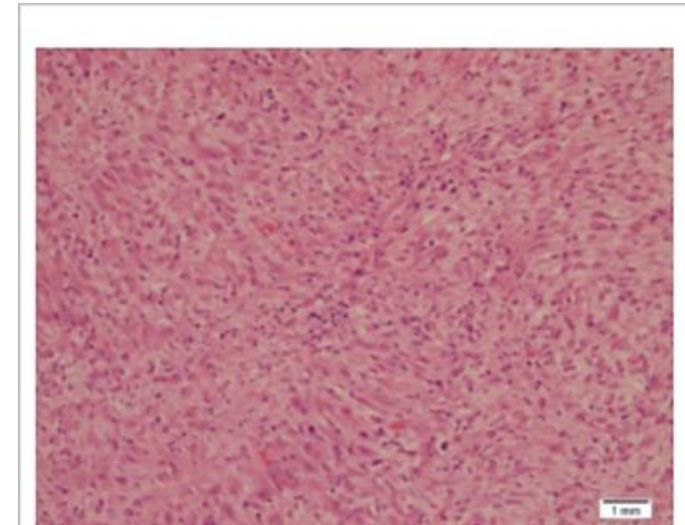

Fig. 5a (10x)

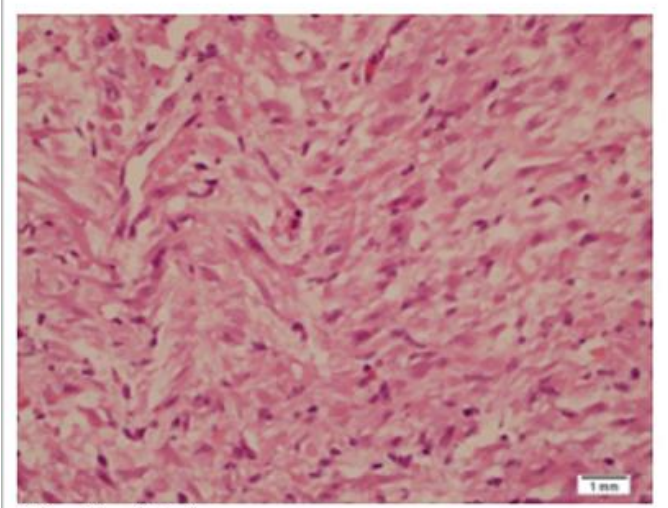

Fig. $5 \mathrm{c}(20 \mathrm{x})$

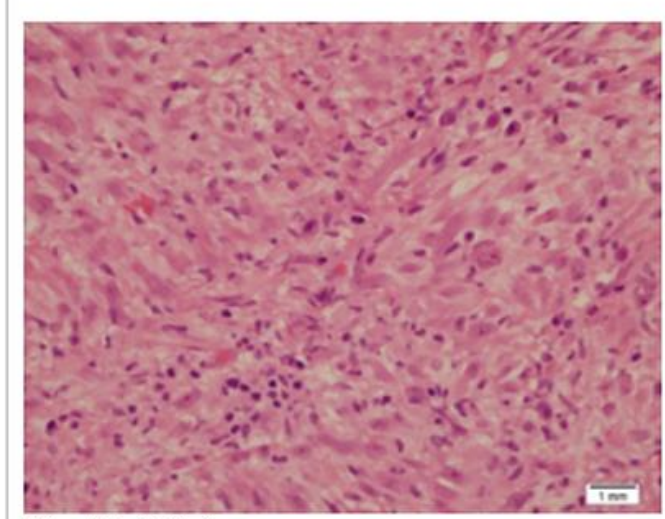

Fig. 5b (20x)

Fig. 5. Microscopic examination of the lesion showed submucosal spindle cells proliferation arranged in a fascicular and storiform pattern (Fig. 4) on a myxoid background with mixed inflammatory cells infiltrate (Fig. 5) composed mainly of lymphocytes, plasma cells along with scattered eosinophils. The lesion abuts on adjacent muscularis propria. Tumor cells frequently have ovoid nuclei with prominent nucleoli (Fig. 6). Mitoses are rare. The Immunohistochemical study (Fig. 7) revealed tumor cells that are diffusely positive for SMA (smooth muscle actin), ALK1 and P-catenin (cytoplasmic), Ki67 5\%. Tumor cells are negative for desmin, CD34 and c-kit. 


\section{Case Reports in Oncology}

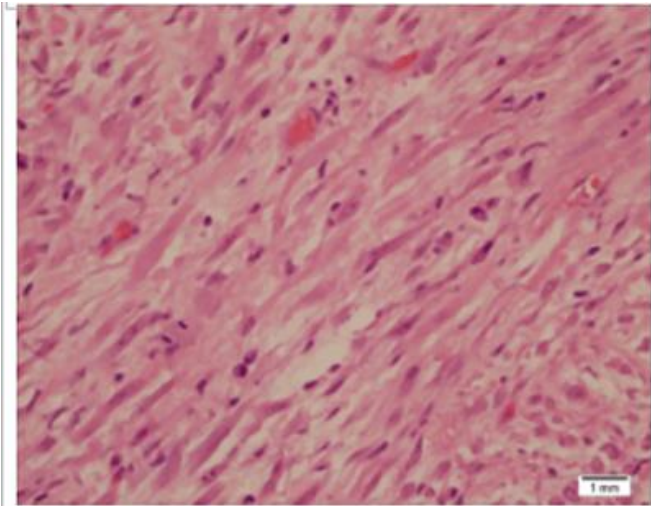

Fig. 6a (40x)

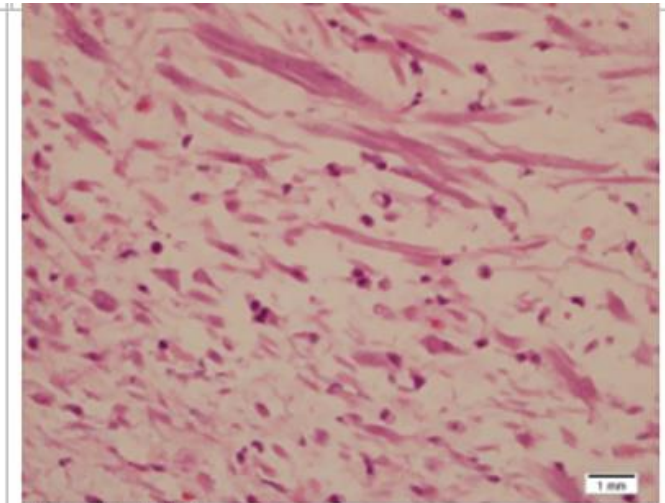

Fig. 6b (40x)

Fig. 6. Microscopic examination of the lesion showed submucosal spindle cells proliferation arranged in a fascicular and storiform pattern (Fig. 4) on a myxoid background with mixed inflammatory cells infiltrate (Fig. 5) composed mainly of lymphocytes, plasma cells along with scattered eosinophils. The lesion abuts on adjacent muscularis propria. Tumor cells frequently have ovoid nuclei with prominent nucleoli (Fig. 6). Mitoses are rare. The Immunohistochemical study (Fig. 7) revealed tumor cells that are diffusely positive for SMA (smooth muscle actin), ALK1 and P-catenin (cytoplasmic), Ki67 5\%. Tumor cells are negative for desmin, CD34 and c-kit. 


\section{Case Reports in Oncology}

\begin{tabular}{l|l}
\hline Case Rep Oncol 2019;12:344-353 \\
\hline DOI: 10.1159/000500503 & $\begin{array}{l}\text { @ 2019 The Author(s). Published by S. Karger AG, Basel } \\
\text { www.karger.com/cro }\end{array}$ \\
\hline
\end{tabular}

Fadaak et al.: Transurethral Resection for the Treatment of an Inflammatory Myofibroblastic Tumor of the Urinary Bladder: A Case Report

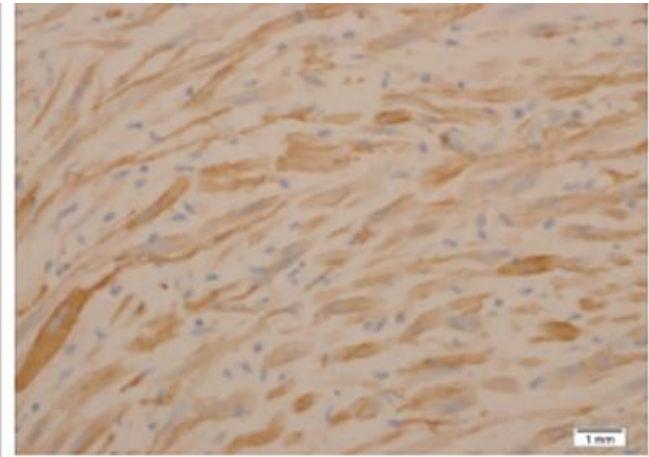

Fig. 7a (SMA)

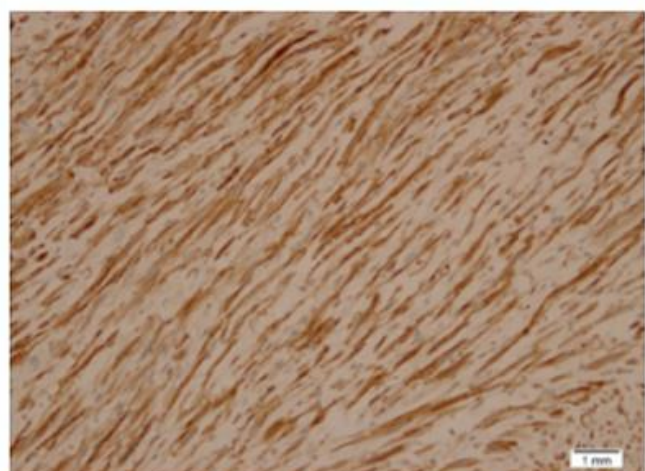

Fig. 7c (P catenin)

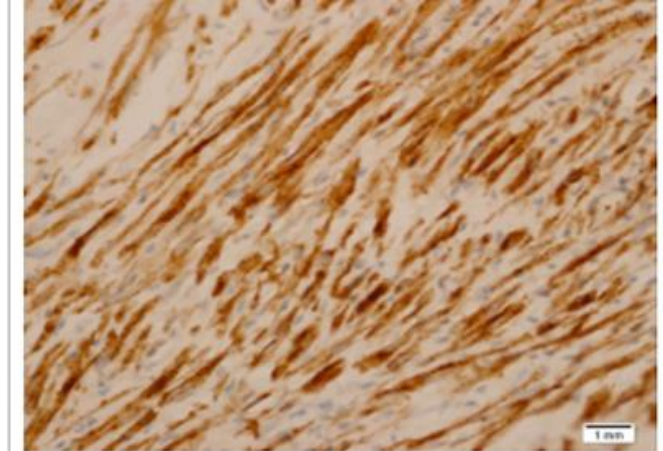

Fig. 7b (ALK1)

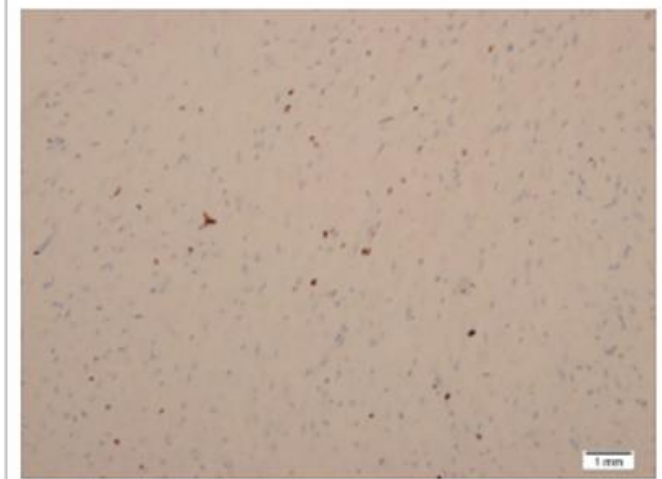

Fig. 7d (Ki67)

Fig. 7. Microscopic examination of the lesion showed submucosal spindle cells proliferation arranged in a fascicular and storiform pattern (Fig. 4) on a myxoid background with mixed inflammatory cells infiltrate (Fig. 5) composed mainly of lymphocytes, plasma cells along with scattered eosinophils. The lesion abuts on adjacent muscularis propria. Tumor cells frequently have ovoid nuclei with prominent nucleoli (Fig. 6). Mitoses are rare. The Immunohistochemical study (Fig. 7) revealed tumor cells that are diffusely positive for SMA (smooth muscle actin), ALK1 and P-catenin (cytoplasmic), Ki67 5\%. Tumor cells are negative for desmin, CD34 and c-kit. 